\title{
One-year Results of Aflibercept Treatment for Polypoidal Choroidal Vasculopathy with Good Visual Acuity
}

\author{
Ji Min Kwon ${ }^{1}$, Kang Yeun Pak ${ }^{1}$, Jae Jung Lee ${ }^{2,3}$, Min Sagong ${ }^{4}$, Hyun Woong Kim ${ }^{1}$ \\ ${ }^{1}$ Department of Ophthalmology, Haeundae Paik Hospital, Inje University College of Medicine, Busan, Korea \\ ${ }^{2}$ Department of Ophthalmology, Pusan National University School of Medicine, Yangsan, Korea \\ ${ }^{3}$ Biomedical Research Institute, Pusan National University Hospital, Busan, Korea \\ ${ }^{4}$ Department of Ophthalmology, Yeungnam University College of Medicine, Daegu, Korea
}

Purpose: To evaluate the one-year efficacy of intravitreal aflibercept injection in polypoidal choroidal vasculopathy (PCV) with best-corrected visual acuity (BCVA) of 20 / 40 or better.

Methods: This was a multicenter retrospective study. The medical records of patients diagnosed with treatment-naïve PCV were retrospectively reviewed. Patients with an initial BCVA of 20 / 40 or better and who had undergone intravitreal aflibercept injection were included. Patients were treated with three consecutive monthly injections, followed by pro re nata regimen according to the clinician's discretion at variable interval visits. The proportions of eyes for which BCVA was maintained ( $\leq 0.2$ logarithm of the minimum angle of resolution change) or improved at 12 months were evaluated. The changes of BCVA, central subfield macular thickness (CSMT), pigment epithelial detachment, and subretinal fluid also were assessed.

Results: A total of 86 eyes were included. The mean number of injections for 12 months of treatment was 5.4 \pm 1.7 . BCVA was maintained or improved in 94.2\% (81 / 86) of cases. Mean BCVA (logarithm of the minimum angle of resolution) had changed from the baseline $(0.23 \pm 0.09)$ at 3 months $(0.21 \pm 0.14), 6$ months $(0.24 \pm 0.22)$, and 12 months (0.20 $\pm 0.18)$, but with no statistical significance. CSMT had improved significantly from the baseline $(336.1 \pm 97.3 \mu \mathrm{m})$ at 3 months $(223.6 \pm 47.22 \mu \mathrm{m}), 6$ months $(239.6 \pm 64.2 \mu \mathrm{m})$, and 12 months $(223.8 \pm 47.9 \mu \mathrm{m})$. Pigment epithelial detachment was observed in $93 \%$ of cases at the baseline, $72.1 \%$ at 3 months, and $69.8 \%$ at 12 months, showing a significant decrease at all observation points. Subretinal fluid was observed in $91.9 \%$ of cases at the baseline, $20.9 \%$ at 3 months, and $29.1 \%$ at 12 months, showing a significant decrease at all observation points.

Conclusions: In cases of PCV with good visual acuity, intravitreal aflibercept injections decreased CSMT and were effective in maintaining visual acuity.

Key Words: Aflibercept, Intravitreal injections, Polypoidal choroidal vasculopathy

Received: July 31, 2020 Final revision: November 5, 2020 Accepted: November 6, 2020

Corresponding Author: Hyun Woong Kim, MD, PhD. Department of Ophthalmology, Haeundae Paik Hospital, Inje University College of Medicine, 875 Haeundae-ro, Haeundae-gu, Busan 48108, Korea. Tel: 8251-797-2310, Fax: 82-51-797-2321, E-mail: maekbakhp@paik.ac.kr
Polypoidal choroidal vasculopathy (PCV) is a type of neovascular age-related macular degeneration (AMD) that is characterized by a network of branching choroidal vessels with terminal, polyp-like aneurysmal dilations [1,2]. PCV is more prevalent in the East than in the West. It ac- 
counts for up to $54.7 \%$ of neovascular AMD in Asians, $22.2 \%$ to $36.3 \%$ in South Koreans, while only about $8 \%$ to $13 \%$ in Caucasians [3-8]. The natural course and response to treatment often differ from typical neovascular AMD. PCV usually causes fewer fibrotic scars, progresses slowly, and shows a favorable prognosis relative to typical neovascular AMD $[2,9]$. However, it often causes persistent bleeding and exudative changes, leading to a chronic and recurrent course that ends in poor visual prognosis due to retinal pigment epithelial (RPE) atrophy $[10,11]$.

Photodynamic therapy (PDT) is an effective treatment for closure of polypoidal lesions but is less effective for regression of branching vascular networks; indeed, only limited improvement of visual acuity has been reported from long term follow-up studies $[12,13]$. Monotherapy with ranibizumab (Lucentis; Genentech, South San Francisco, CA, USA), an anti-vascular endothelial growth factor (anti-VEGF) monoclonal antibody, has effected significant visual improvement [14] but only a limited polyp regression rate of $25 \%$ to $40 \%$ [15-18]. Aflibercept (Eylea; Regeneron, Tarrytown, NY, USA), a recombinant fusion protein of anti-VEGF, has been reported to have similar efficacy and safety to ranibizumab with longer half-time in neovascular AMD, and there have been reports of the administration of intravitreal aflibercept injections for PCV as well. In fact, many studies have noted visual and anatomical improvements associated with intravitreal aflibercept injections, but in most cases, the initial best-corrected visual acuity (BCVA) was worse than 20 / 40 [19,20].

While many studies have been reported using aflibercept in AMD with good visual acuity, there were few studies only on PCV patients. In addition, the number of PCV patients was small in studies of AMD patients including PCV. The purpose of the present study was to evaluate the 1-year efficacy of intravitreal aflibercept injections for PCV in eyes with BCVA of $20 / 40$ or better.

\section{Materials and Methods}

This was a multi-center retrospective observational study that adhered to the tenets of the Declaration of Helsinki and was approved by the institutional review board of Inje University Haeundae Paik Hospital (2019-03-016001). Written informed consent was waived due to the retrospective nature of the study. We retrospectively reviewed the medical records of patients with PCV who had received intravitreal aflibercept injections and been followed up for 12 months between January 2014 and December 2018 at Inje University Haeundae Paik Hospital, Pusan National University Hospital, Pusan National University Yangsan Hospital, and Yeungnam University Medical Center. The diagnosis of PCV, made by indocyanine green angiography, was based primarily on findings of polypoidal hyperfluorescent lesions and branching vascular networks, and was confirmed when intraretinal or subretinal fluid (SRF) was accompanied on optical coherence tomography (OCT). The study-inclusion criteria were initial BCVA of $20 / 40$ or better, and no change of treatment drug for at least 1 year after the initial administration. The exclusion criteria were as follows: previous history of intravitreal anti-VEGF or PDT; severe media opacity, previous history of vitreoretinal surgery, proliferative diabetic retinopathy, retinal vascular occlusion, macular hole, or any other retinal disorder that could influence macular microstructure and/or function.

At baseline, patients' history of systemic and ophthalmic diseases was checked prior to initiation of treatment. BCVA was measured on the Snellen visual acuity chart. Slit-lamp microscopy, fundus examinations, fundus photography, OCT, and fluorescein and indocyanine green angiography were performed. Fundus photographs and OCT images were obtained according to each center's setting (including TRC-NW8 [Topcon, Tokyo, Japan], Nonmyd7 [Kowa, Tokyo, Japan], TRC-50DX [Topcon] for fundus photographs and Cirrus HD-OCT [Carl Zeiss Meditec, Dublin, CA, USA], DRI OCT-1 [Topcon] for OCT images). All hospitals used Spectralis HRA (Heidelberg Engineering, Heidelberg, Germany) for fluorescein and indocyanine green angiography. BCVA and OCT were measured at 3,6, 9 , and 12 months after treatment.

All of the patients were given an initial three loadings of intravitreal aflibercept ( $2.0 \mathrm{mg}$ in $0.05 \mathrm{~mL}$ ) at 1-month intervals. After the 3 loading injections, follow-up visits were performed every 1 to 3 months, according to the clinician's judgment. BCVA, fundus examinations, fundus photography, and OCT also were checked or performed at each visit. Additional injections were performed on an "as-needed" basis (i.e., in cases where OCT-detected subretinal or intraretinal fluid (IRF) threatened the fovea, or retinal or subretinal hemorrhage involved the macula) (Fig. 1).

The BCVA was converted to the logarithm of minimal 


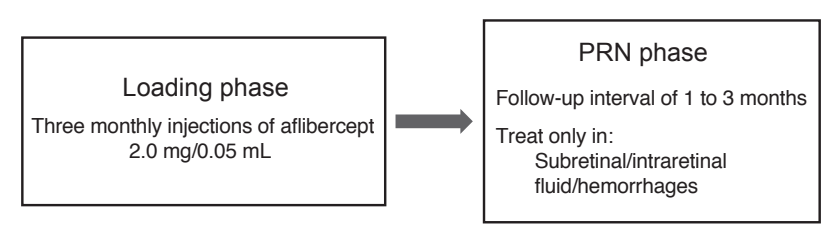

Fig. 1. Schematic flow chart of the treatment regimen using intravitreal aflibercept injections for the treatment of polypoidal choroidal vasculopathy. $\mathrm{PRN}=$ pro re nata .

angle of resolution (logMAR) scale for analysis. BCVA 3, 6,9 , and 12 months after treatment were compared with the baseline, and the proportion of eyes that maintained or improved visual acuity was evaluated. The change of BCVA was classified as improvement, maintenance and worsen with a cut-off value of $0.2 \log$ MAR [21]. Central subfield macular thickness (CSMT) 3, 6, 9, and 12 months after treatment were compared with the baseline, as were changes of pigment epithelial detachment (PED), SRF, and IRF. CSMT was defined as the average retinal thickness in the 1-mm diameter center of the fovea. PED was defined as an elevation of the RPE layer over Bruch's membrane, SRF was defined as a hyporeflective space between neurosensory retina and RPE. Both were assessed based on the OCT line scans of fovea, and only cases that violated the fovea were counted.

Changes in BCVA and CSMT from the baseline were assessed by Wilcoxon signed-rank test. The proportions of eyes that maintained or improved BCVA at 3 and 12 months after treatment, and the proportions of eyes with PED, SRF, and IRF at 3, 6, and 12 months after treatment, were compared by McNemar test. The visual outcomes were analyzed using Mann-Whitney $U$-test by subgrouping patients according to baseline BCVA, baseline CSMT, presence of SRF at 3 months, and total injection numbers. To analyze factors affecting final BCVA, multiple linear regression analysis was performed. The analyses were conducted using IBM SPSS Statistics ver. 22.0 (IBM Corp., Armonk, NY, USA), and $p$-values $<0.05$ were considered statistically significant.

\section{Results}

A total of 86 eyes of 86 patients, 60 males and 26 females, were included in the study. The mean age was 65.6 \pm 7.26 years (Table 1 ). The mean number of injections was $5.4 \pm 1.7$. BCVA was maintained or improved in $94.2 \%$ (81 / 86) of cases: $83.7 \%(72 / 86)$ and $10.5 \%$ (9/ 86) improvement. In eyes with maintained BCVA (changes in BCVA $\leq 0.2 \log$ MAR), 12 (14\%) showed a gain of $0.2 \log$ MAR, and $21(24.4 \%)$ a gain of $0.1 \log$ MAR (Fig. 2). The proportion of eyes that maintained or improved BCVA at 12 months after treatment $(94.2 \%)$ was not significantly different from that at 3 months $(97.7 \%)(p=0.371)$.

Table 1. Baseline characteristics of patients

\begin{tabular}{lc}
\hline Variable & Value \\
\hline Age $(\mathrm{yr})$ & $65.6 \pm 7.26(52-81)$ \\
Sex (male : female) & $60: 26$ \\
BCVA (logMAR) & $0.23 \pm 0.09(0.0-0.3)$ \\
CSMT $(\mu \mathrm{m})$ & $336.1 \pm 97.3(139-597)$ \\
PED $(\%)$ & 93.0 \\
SRF $(\%)$ & 91.9 \\
\hline
\end{tabular}

Values are presented as mean \pm standard deviation (range) or number.

$\mathrm{BCVA}=$ best-corrected visual acuity; $\log \mathrm{MAR}=$ logarithm of minimal angle of resolution; CSMT = central subfield macular thickness; PED = pigment epithelial detachment; SRF = subretinal fluid.

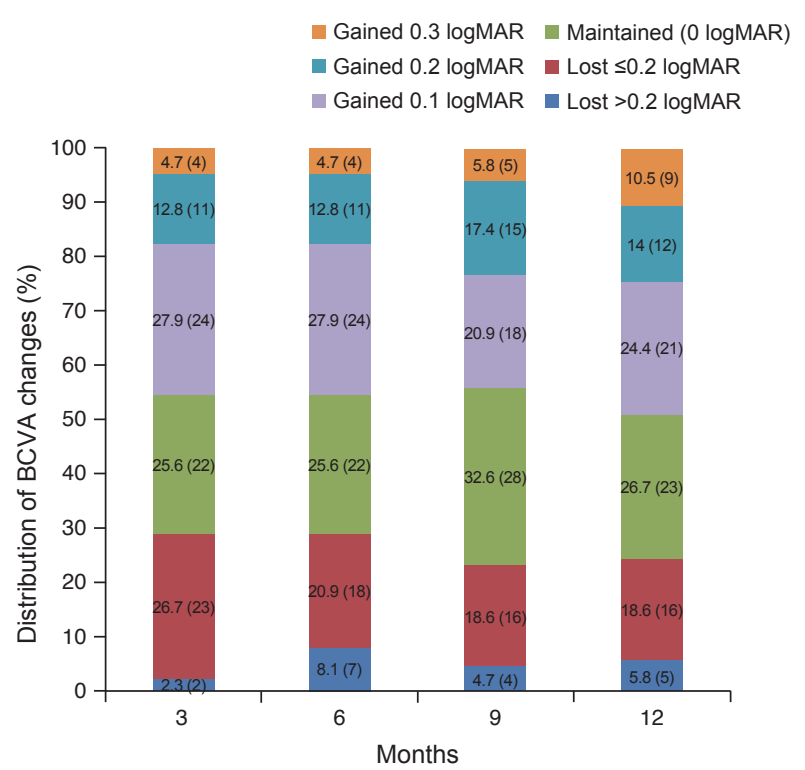

Fig. 2. Distribution of best-corrected visual acuity (BCVA) changes. There was no significant difference in the "improved" or "maintained" proportion between 3 months (97.7\%) and 12 months $(94.2 \%)$. logMAR $=$ logarithm of minimal angle of resolution. 

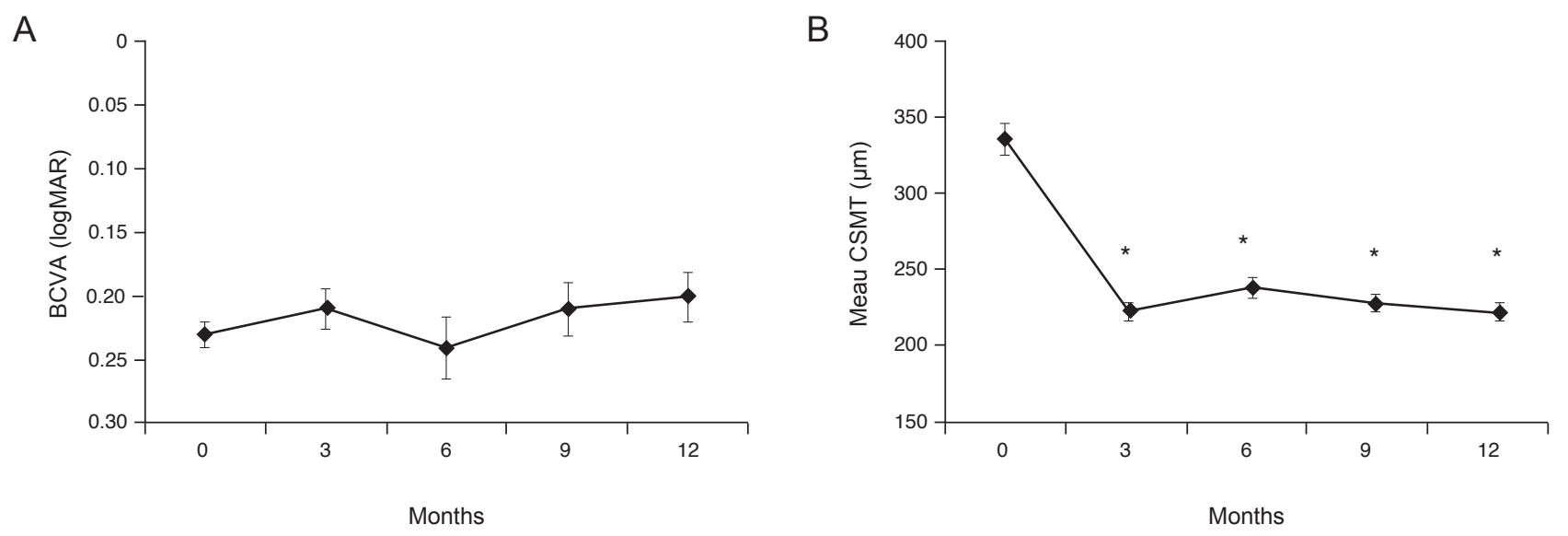

Fig. 3. Mean changes in best-corrected visual acuity (BCVA) and central subfield macular thickness (CSMT) after intravitreal aflibercept injection. (A) Mean BCVA (logMAR) had changed from the baseline $(0.23 \pm 0.09)$ at 3 months $(0.21 \pm 0.14), 6$ months $(0.24 \pm 0.22)$, and 12 months $(0.20 \pm 0.18)$, but with no statistical significance. (B) CSMT had improved significantly from the baseline $(336.1 \pm 97.3 \mu \mathrm{m})$ at 3 months $(223.6 \pm 47.22 \mu \mathrm{m}, p<0.001), 6$ months $(239.6 \pm 64.2 \mu \mathrm{m}, p<0.001)$, and 12 months $(223.8 \pm 47.9 \mu \mathrm{m}, p<0.001)$.

${ }^{*}$ Statistically significant difference from the baseline $(p<0.05)$.

The mean BCVA was $0.23 \pm 0.09,0.21 \pm 0.14,0.24 \pm$ $0.22,0.21 \pm 0.20$, and $0.20 \pm 0.18$ at baseline and $3,6,9$, and 12 months after treatment, respectively (Fig. 3A). There was no significant relative-to-baseline improvement at any of the observation points after treatment $(p=0.116, p=$ $0.544, p=0.080, p=0.068$, respectively). The mean CSMT was $336.1 \pm 97.3,223.6 \pm 47.22,239.6 \pm 64.2,229.4 \pm 47.1$, and $223.8 \pm 47.9 \mu \mathrm{m}$ at the baseline and 3, 6, 9, and 12 months after treatment, respectively (Fig. 3B). Significant improvement was seen at all observation points after treatment relative to the baseline $(p<0.001, p<0.001, p<0.001$, $p<0.001$, respectively).

PED was observed in $93 \%$ ( $80 / 86$ ) of cases at the baseline, $72.1 \%(62 / 86)$ at 3 months, $72.1 \%(62 / 86)$ at 6 months, and $69.8 \%(60 / 86)$ at 12 months. The proportion of PED was significantly decreased at all observation points relative to the baseline ( $p=0.001, p=0.001, p<$ 0.001, respectively) (Fig. 4). SRF was observed in 91.9\% (79 / 86) of cases at the baseline, 20.9\% (18/86) at 3 months, $30.2 \%(26 / 86)$ at 6 months, and 29.1\% (25/86) at 12 months. The proportion of SRF was significantly decreased at all observation points relative to the baseline ( $p$ $<0.001, p<0.001, p<0.001$ ) (Fig. 4). IRF was observed in $20.9 \%(18 / 86)$ of cases at the baseline, $3.5 \%(3 / 86)$ at 3 months, 5.8\% (5 / 86) at 6 months, and 3.5\% (3/ 86) at 12 months. The proportion of SRF was significantly decreased at all observation points relative to the baseline ( $p$ $<0.001, p=0.001, p<0.001$ ) (Fig. 4).

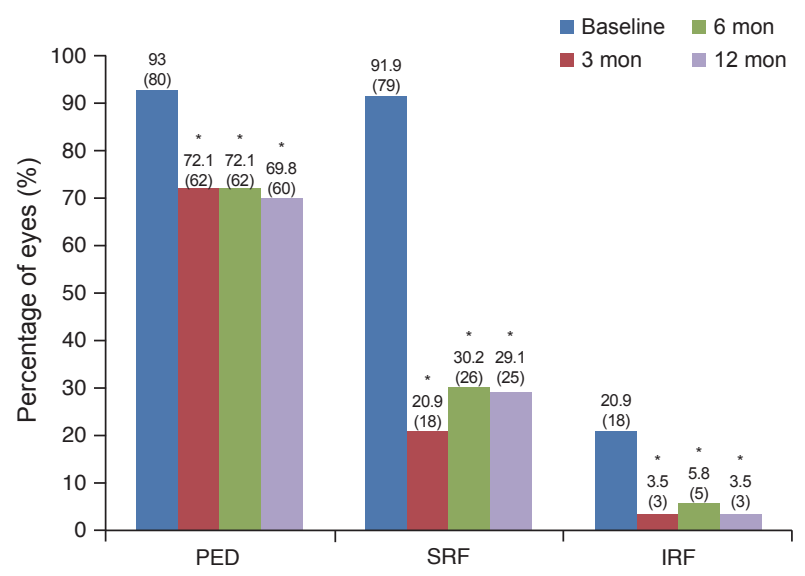

Fig. 4. Proportions of eyes with pigment epithelial defect (PED), subretinal fluid (SRF), and intraretinal fluid (IRF). The proportions of PED, SRF, and IRF were significantly decreased at all observation points relative to the baseline. "Statistically significant difference from the baseline $(p<0.05)$.

Final visual outcomes were analyzed by subgrouping patients according to baseline BCVA, baseline CSMT, presence of SRF at 3 month, and total injection numbers (Fig. 5). The patients with baseline BCVA of $0.2 \log$ MAR or better had significantly better final BCVA than the patients with baseline BCVA worse than $0.2 \log$ MAR (Fig. 5A). Stratification based on baseline CSMT of $300 \mu \mathrm{m}$ showed a significant difference in BCVA only before treatment, and no significant difference was observed during the study period (Fig. 5B). The group with SRF at 3 
A

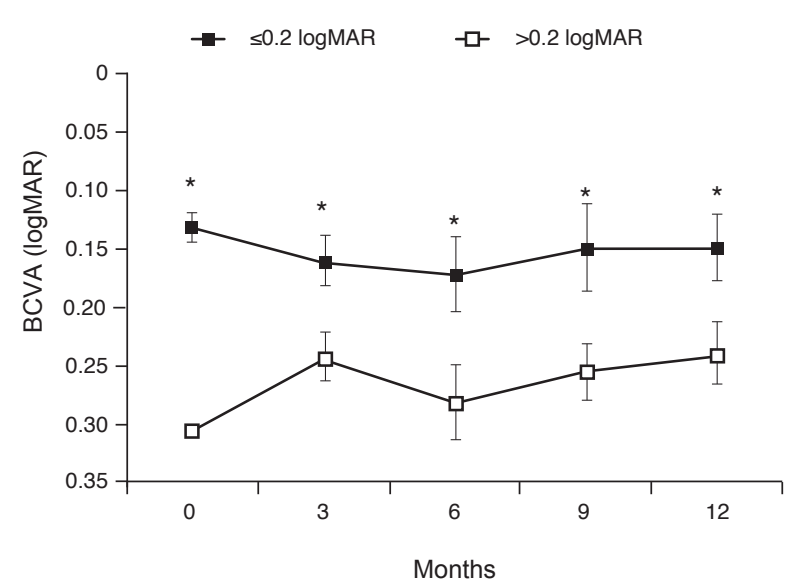

C

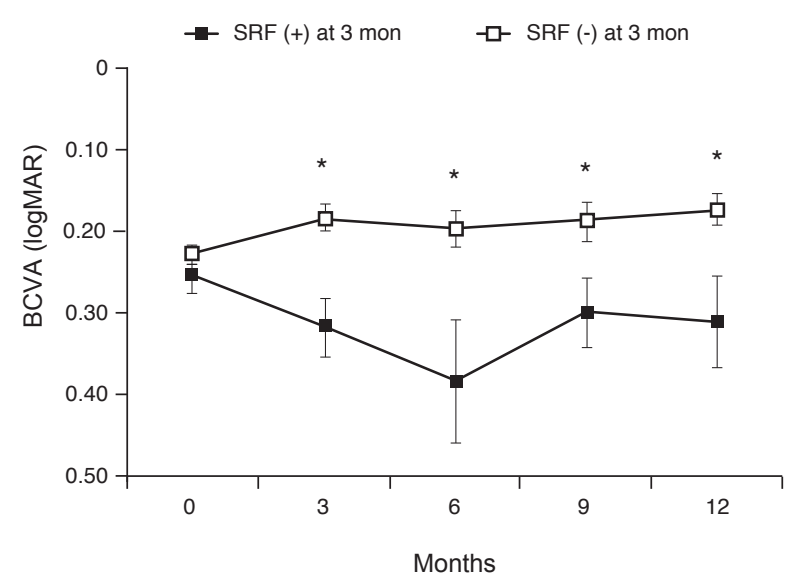

B

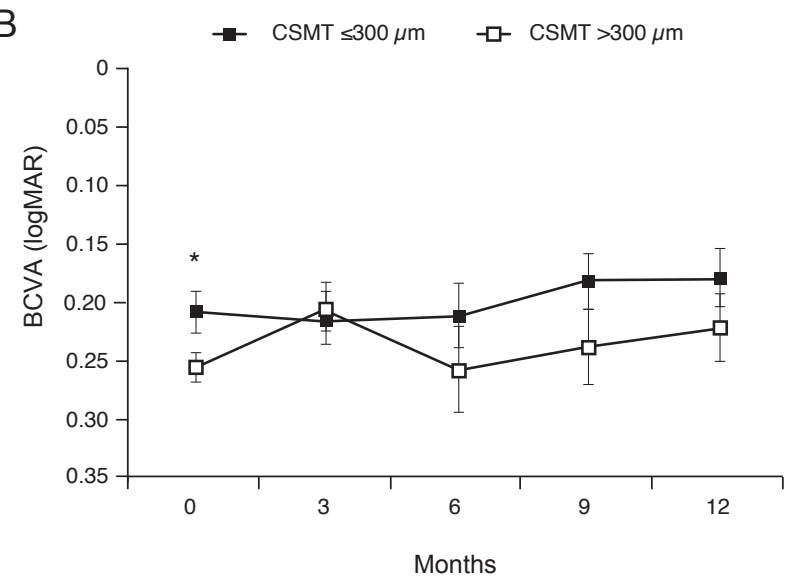

D

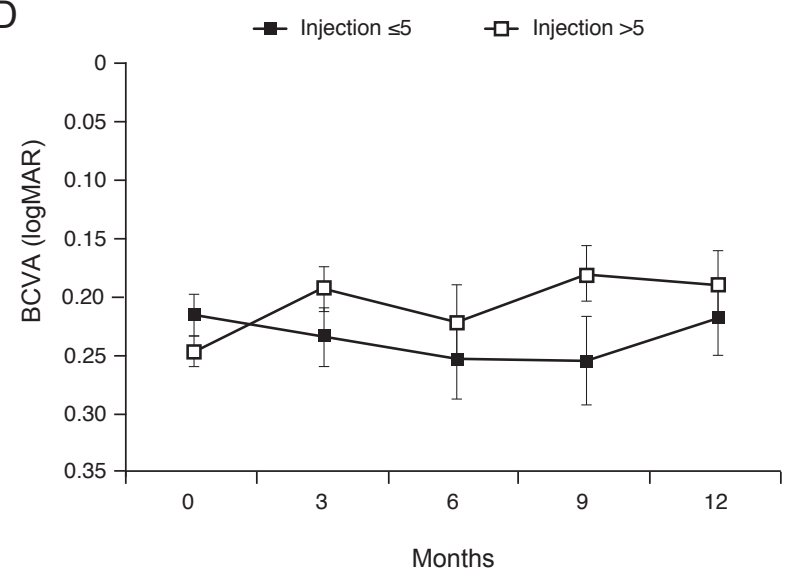

Fig. 5. Subgroup analysis according to baseline best-corrected visual acuity (BCVA), baseline central subfield macular thickness (CSMT), presence of subretinal fluid (SRF) at 3 month, and total injection numbers. (A) Eyes with baseline BCVA of 0.2 logarithm of minimal angle of resolution ( $\log$ MAR) or better had significantly better final BCVA. (B) Stratification based on baseline CSMT of $300 \mu \mathrm{m}$ showed a significant difference in BCVA only before treatment, and no significant difference was observed during the study period. (C) Eyes with SRF at 3 months had significantly worse final BCVA. (D) Stratification according to the number of injections showed no significant difference in BCVA during the entire study period. "Statistically significant difference from the baseline $(p<0.05)$.

months had significantly worse final BCVA (Fig. 5C), and stratification according to the number of injections showed no significant difference in BCVA (Fig. 5D). Similarly, in a multiple linear regression analysis, baseline BCVA and $\mathrm{SRF}$ at 3 months were found to affect final BCVA (Table 2).

A total of 466 injections were performed over 12 months, with no serious adverse events such as endophthalmitis or retinal detachment. Five eyes showed decreased BCVA despite treatment, due to a submacular hemorrhage in one eye and macular atrophy in the other four.

A representative case is shown in Fig. 6A-6E. A 62-yearold man diagnosed with PCV maintained good visual acu-
Table 2. Multiple linear regression analysis for final visual acuity

\begin{tabular}{lcc}
\hline Variable & $\beta$ coefficient & $p$-value \\
\hline Age (yr) & 0.098 & 0.357 \\
Sex, male (reference: female) & 0.202 & 0.052 \\
$\begin{array}{l}\text { Baseline BCVA (logMAR) } \\
\begin{array}{l}\text { SRF at 3 months, presence } \\
\text { (reference: absence) }\end{array}\end{array}$ & 0.213 & $0.047^{*}$ \\
No. of injections & 0.267 & $0.020^{*}$ \\
\hline
\end{tabular}

$\mathrm{BCVA}=$ best-corrected visual acuity; $\log \mathrm{MAR}=$ logarithm of minimal angle of resolution; $\mathrm{SRF}=$ subretinal fluid.

"Statistically significant difference from the baseline $(p<0.05)$. 

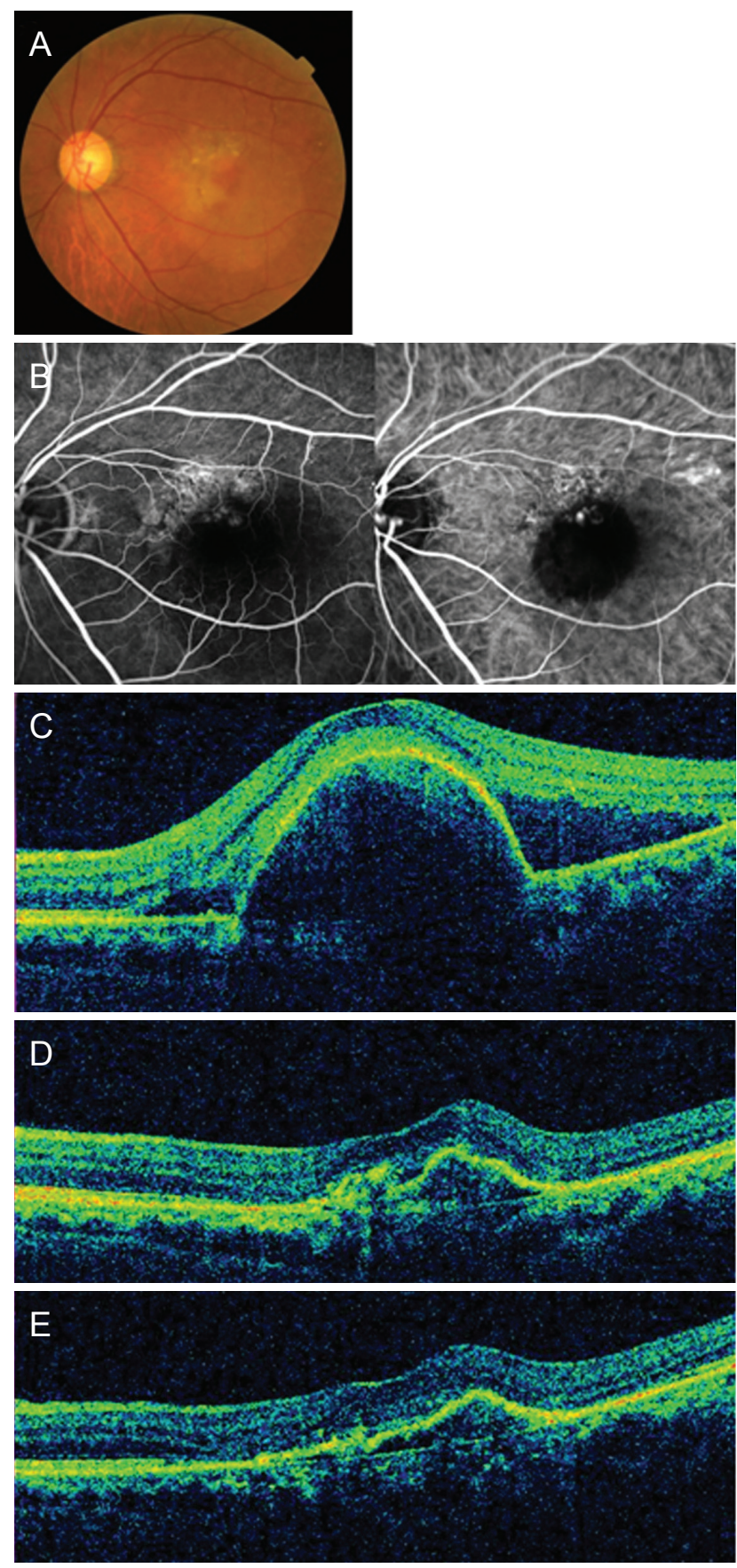

Fig. 6. A representative case of a 62-year-old man with polypoidal choroidal vasculopathy treated with intravitreal aflibercept injections. (A) Baseline color fundus photograph shows orange polypoidal lesion and large area of subretinal fluid (SRF). (B) Baseline fluorescein and indocyanine green angiography shows polys and a network of abnormal vessels. (C) Baseline optical coherence tomography image shows large pigment epithelial detachment and SRF related to polypoidal choroidal vasculopathy. Visual acuity was $20 / 40$. (D) After 3 monthly loading phase, SRF had resolved, but polypoidal lesions persisted. Visual acuity was $20 / 25$. (E) At 1 year after the first treatment, the patient's visual acuity was $20 / 40$. In total, seven intravitreal aflibercept treatments were administered during the follow-up period. ity of 20 / 40 with a total of seven intravitreal aflibercept injections.

\section{Discussion}

PCV and neovascular AMD share similarities in their association with choroidal neovascularization and genetic variations, but they also show some differences [13,14]. Choroidal thickness is thicker in PCV, and fibrotic progression is more common in neovascular AMD. Although the expression level of VEGF is increased in both PCV and neovascular AMD as compared with healthy eyes, the VEGF level in eyes with PCV is considerably lower than in AMD eyes [22,23]. There has also been an increase in inflammatory cytokines, including interleukin-1 $\beta$ and interleukin-23, in the anterior chamber and vitreous bodies, suggesting that they play an inflammatory-response role in PCV $[24,25]$. These differences might be among the reasons why the therapeutic response of anti-VEGF agents in $\mathrm{PCV}$ is not as good as in typical neovascular AMD.

Treatment strategy for PCV varies according to studies. PDT causes polyp regression by selectively closing neovascularization, and was widely used for PCV before the development of anti-VEGF agents. However, as it was less effective in the closure of branching vascular networks than for polyps, limitations in visual improvement were identified in reports with long follow-up periods [12]. Rare but serious complications, such as subretinal hemorrhage, choroidal infarction, and RPE rupture, also have been reported [26-28]. After confirmation of the effects of ranibizumab on neovascular AMD in large clinical studies, intravitreal anti-VEGF injection has replaced PDT as the primary treatment for PCV [29,30]. Early studies reported that ranibizumab monotherapy showed an effective visual improvement [15], but the rate of polyp closure was low, ranging from $25 \%$ to $40 \%$ [16-18,31]. In the EVEREST study comparing the efficacy of PDT monotherapy, ranibizumab monotherapy, and a PDT/ranibizumab combination therapy, the combination therapy and PDT monotherapy were superior to ranibizumab monotherapy in achieving complete regression of polyps at 6 months and 12 months, and improvement in BCVA at 12 months [19,32]. However, in the LAPTOP study comparing the effects of PDT and ranibizumab monotherapy, ranibizumab monotherapy was better than PDT in PCV patients, and possible damage to 
the RPE and photoreceptors by PDT could account for the results [21]. Aflibercept binds not only to VEGF-A but also to VEGF-B and placental growth factor with greater affinity, and thus, it was expected to be more effective than bevacizumab (Avastin, Genentech) or ranibizumab, both of which bind only to VEGF-A [33]. Cho et al. [34] compared the effectiveness of intravitreal injection of aflibercept and ranibizumab for patients with PCV. The mean number of injections for 1 year did not differ between the two groups $(p=0.152)$. After 12 months of injection, there was no significant difference between the groups concerning either BCVA improvement or decreased central foveal thickness, though polyp regression was significantly more frequent in the aflibercept-treated group.

The therapeutic effects of ranibizumab have been reported for eyes with good initial BCVA. In 2012, Saito et al. [35] performed an average of 4.9 injections of intravitreal ranibizumab in $18 \mathrm{PCV}$ eyes with a baseline BCVA exceeding 20 / 40, and reported a significant improvement in BCVA from the baseline (0.17 $\log$ MAR) to 12 months (0.07 logMAR). Mori et al. [36] performed an average of 4.7 injections of intravitreal ranizbizumab in $50 \mathrm{PCV}$ eyes with a baseline BCVA of 0.6 to 1.0, and reported a significant improvement in BCVA from the baseline (0.11 logMAR) to 12 months (0.07 logMAR). Kato et al. [37] administered intravitreal ranibizumab in PCV with baseline BCVA of $0.10 \operatorname{logMAR}$. With 3.05 injections, the absolute value of visual acuity improved to 0.09 , but there was no statistical significance.

In most studies of intravitreal aflibercept injections for PCV in Korea, the initial BCVA is less than $20 / 40$, and eyes with good visual acuity of 20 / 40 or better are not included [20,34,38]. Cho et al. [34] showed significant improvement in visual acuity at 12 months (0.44 $\log$ MAR) relative to the baseline ( $0.63 \log \mathrm{MAR})$ after intravitreal aflibercept injections in $38 \mathrm{PCV}$ eyes. Kim et al. [38] reported a retrospective review of $46 \mathrm{PCV}$ eyes treated with intravitreal aflibercept monotherapy for 24 months. An average of 4.7 injections was administered in the first 12 months, and 2.3 injections in the second 12 months. BCVA was significantly improved at 12 months relative to the baseline values. At 24 months there was no significant difference, but BCVA was maintained or improved in $76.1 \%$ of cases. In the VAULT study, Lee et al. [20] administered seven injections of intravitreal aflibercept in 40 PCV eyes for 1 year (at 2-month intervals after loading doses), and found significant improvement in visual acuity at 12 months (20 / 53) relative to the baseline (20 / 80). In Japan, Oshima et al. [39] performed 2-month interval fixed treatments after loading doses of aflibercept in 50 PCV eyes. BCVA had significantly improved at 12 months (0.12 logMAR) compared to the baseline (0.33 logMAR). However, no independent analysis of eyes with good initial vision was performed in any of the above reports.

The effects of intravitreal aflibercept injection in PCV patients with good visual acuity have been reported in Japanese studies. Minami et al. [40] injected aflibercept in AMD patients with initial visual acuity greater than 0.6 , and revealed that there was significant improvement in BCVA at 6 months (0.03 logMAR) and 12 months (0.04 $\log$ MAR) compared to baseline (0.07 logMAR). Of the 29 eyes, 17 PCV eyes were included, but no subgroup analysis was implemented for PCV. Sakamoto et al. [41] injected aflibercept in AMD patients with initial visual acuity greater than 0.5 (typical AMD 18 eyes and PCV 21 eyes). While BCVA improved significantly at 12 months (0.06 logMAR) compared to baseline (0.10 $\log$ MAR) for the entire study group, PCV eyes showed a lack of significance compared to the baseline $(0.07 \log$ MAR) in 12 months $(0.05$ logMAR). Although the above two studies included PCV with good visual acuity in the study population, the number of PCV patients was not large enough for subgroup analysis. This is different from our study, which was conducted exclusively with PCV patients.

In the present study, BCVA increased from 0.23 logMAR (at baseline) to $0.20 \log$ MAR (12 months) with an average of 5.4 intravitreal aflibercept injections. However, the values were not significantly different relative to the baseline. In reports on the utilization of the pro re nata method, the number of injections of aflibercept and ranibizumab for PCV with poor visual acuity averaged, comparably to the present study, 4.7 to 4.9 over the course of 12 months [34-36,38]. There was no significant difference in the number of injections in 12 months between studies on good-visual-acuity and poor-visual-acuity eyes. The reason seems to be that most studies are conducted based on 3 monthly loading injections, making it difficult to show difference between the remaining 9 months due to the recommended 2-month-interval administration. More long-term studies are needed for more meaningful comparisons.

Although this study did not show significant visual improvement at 12 months after treatment, the change of vi- 
Table 3. Comparison of the 1-year results of the current study to previous studies

\begin{tabular}{|c|c|c|c|c|c|c|c|c|c|c|}
\hline Study & $\begin{array}{c}\text { Total } \\
\text { sample size } \\
\text { (PCV } \\
\text { subgroup) }\end{array}$ & Ethnicity & Regimen & Drug & $\begin{array}{c}\text { Included } \\
\text { only } \\
\text { good } \\
\text { baseline } \\
\text { BCVA }\end{array}$ & $\begin{array}{c}\text { Baseline } \\
\text { BCVA } \\
\text { (logMAR) }\end{array}$ & $\begin{array}{c}\text { BCVA } \\
\text { at } 12 \\
\text { months } \\
(\log \text { MAR) }\end{array}$ & $\begin{array}{l}\text { 12-month } \\
\text { BCVA } \\
\text { change } \\
\text { (logMAR) }\end{array}$ & $\begin{array}{l}\text { No. of } \\
\text { injection }\end{array}$ & $\begin{array}{l}\text { Follow-up } \\
\text { interval } \\
\text { (at PRN } \\
\text { treatment) }\end{array}$ \\
\hline $\begin{array}{l}\text { Saito et al., } \\
2012 \\
{[35]}\end{array}$ & 40 (18) & Japanese & $\begin{array}{c}3 \text { Loadings } \\
+ \\
\text { PRN }\end{array}$ & $\begin{array}{c}\text { Ranibizumab } \\
0.5 \mathrm{mg}\end{array}$ & Yes & 0.14 & $0.07^{*}$ & 0.07 & 4.9 & 1 mon \\
\hline $\begin{array}{l}\text { Mori et al., } \\
2013 \\
{[36]}\end{array}$ & $50(50)$ & Japanese & $\begin{array}{c}3 \text { Loadings } \\
+ \\
\text { PRN }\end{array}$ & $\begin{array}{c}\text { Ranibizumab } \\
0.5 \mathrm{mg}\end{array}$ & Yes & 0.11 & $0.07^{*}$ & 0.04 & 4.72 & $1 \mathrm{mon}$ \\
\hline $\begin{array}{l}\text { Kato et al., } \\
2015 \\
{[37]}\end{array}$ & $36(17)$ & Japanese & $\begin{array}{c}\text { PRN } \\
\text { without } \\
\text { loading }\end{array}$ & $\begin{array}{c}\text { Ranibizumab } \\
0.5 \mathrm{mg}\end{array}$ & Yes & 0.10 & 0.09 & 0.01 & 3.05 & $1 \mathrm{mon}$ \\
\hline $\begin{array}{l}\text { Cho et al., } \\
2016 \\
{[34]}\end{array}$ & $98(60)$ & Korean & $\begin{array}{c}3 \text { Loadings } \\
+ \\
\text { PRN }\end{array}$ & $\begin{array}{c}\text { Ranibizumab } \\
0.5 \mathrm{mg}\end{array}$ & No & 0.66 & $0.49^{*}$ & 0.17 & 5.52 & 1 mon \\
\hline $\begin{array}{l}\text { Cho et al., } \\
2016 \\
{[34]}\end{array}$ & $98(38)$ & Korean & $\begin{array}{c}3 \text { Loadings } \\
+ \\
\text { PRN }\end{array}$ & $\begin{array}{l}\text { Aflibercept } \\
2.0 \mathrm{mg}\end{array}$ & No & 0.63 & $0.44^{*}$ & 0.19 & 4.94 & 1 mon \\
\hline $\begin{array}{l}\text { Lee et al., } \\
2017 \\
{[20]}\end{array}$ & $40(40)$ & Korean & $\begin{array}{c}3 \text { Loadings } \\
+ \\
2 \text { mon }\end{array}$ & $\begin{array}{l}\text { Aflibercept } \\
2.0 \mathrm{mg}\end{array}$ & No & 0.60 & $0.42^{*}$ & 0.18 & 7 & \\
\hline $\begin{array}{l}\text { Oshima et } \\
\text { al., } 2017 \\
\text { [39] }\end{array}$ & $50(50)$ & Japanese & $\begin{array}{c}3 \text { Loadings } \\
+ \\
2 \text { mon }\end{array}$ & $\begin{array}{l}\text { Aflibercept } \\
2.0 \mathrm{mg}\end{array}$ & No & 0.33 & $0.12^{*}$ & 0.21 & 7 & \\
\hline $\begin{array}{l}\text { Kim et al., } \\
2018 \\
{[38]}\end{array}$ & $46(46)$ & Korean & $\begin{array}{c}3 \text { Loadings } \\
+ \\
\text { PRN }\end{array}$ & $\begin{array}{l}\text { Aflibercept } \\
2.0 \mathrm{mg}\end{array}$ & No & 0.56 & $0.45^{*}$ & 0.11 & 4.7 & $1-2$ mon \\
\hline $\begin{array}{c}\text { Sakamoto et } \\
\text { al., } 2018 \\
{[41]}\end{array}$ & $39(21)$ & Japanese & $\begin{array}{c}3 \text { Loadings } \\
+ \\
2 \text { mon }\end{array}$ & $\begin{array}{l}\text { Aflibercept } \\
2.0 \mathrm{mg}\end{array}$ & Yes & 0.07 & 0.05 & 0.02 & 7 & \\
\hline $\begin{array}{r}\text { Current } \\
\text { study }\end{array}$ & $86(86)$ & Korean & $\begin{array}{c}3 \text { Loadings } \\
+ \\
\text { PRN }\end{array}$ & $\begin{array}{l}\text { Aflibercept } \\
2.0 \mathrm{mg}\end{array}$ & Yes & 0.22 & 0.19 & 0.03 & 5.2 & 1-3 mon \\
\hline
\end{tabular}

$\mathrm{PCV}=$ polypoidal choroidal vasculopathy; $\mathrm{BCVA}=$ best-corrected visual acuity; logMAR = logarithm of minimal angle of resolution; $\mathrm{PRN}=$ pro re nata .

${ }^{*}$ Statistically significant difference from the baseline $(p<0.05)$.

sual acuity was comparable to similar study by Mori et al. [36]. And whereas this study adopted the same pro re nata administration, no significant visual improvement was shown, unlike the reports of Saito et al. [35]'s and Mori et al. [36]'s, which showed significant visual improvement by implementation of ranibizumab for PCV with good visual acuity. The reason seems to be baseline BCVA and follow-up intervals. In the current study, a subgroup analysis showed that final visual outcome was poor when baseline BCVA was worse than 0.2 logMAR, and the multiple linear regression analysis showed that final BCVA was asso- ciated with baseline BCVA. The average baseline BCVA of our study was $0.22 \log$ MAR, while those of Saito et al. [35] and Mori et al. [36] were 0.14 and 0.11 , respectively. Therefore, the fact that initial BCVA in this study was worse than that of the previous studies suggests that the disease was relatively in advanced state compared to the previous studies, and the prognosis after treatment may have been worse. As for follow-up intervals, both of those previous studies had follow-up examinations monthly, while in the current study, follow-up visits were scheduled only every 1 to 3 months. Therefore, recurrence could have 
been detected late, which would have resulted in delayed administration. However, similar to current study, Sakamoto et al., who injected intravitreal aflibercept in PCV with good visual acuity, showed no significant improvement in BCVA at 12 months [41]. The mean change of logMAR BCVA they reported was similar to our study.

In earlier studies on PCV with poor visual acuity, the mean BCVA change at 12 months after treatment was 0.11 to $0.19 \log$ MAR, which was greater than in the present study. This is judged to have been due to the ceiling effect, by which, in the current study, initial vision was relatively better and therefore had a lower potential for improvement. However, the final BCVA at 12 months after treatment was $0.20 \log$ MAR, which was higher than in studies on eyes with poor initial visual acuity ( 0.42 to $0.45 \log$ MAR) $[20,34,38]$. The size of lesions would be smaller, the activity of the disease would be less severe, damaged retinal tissues that cannot be recovered would be less prevalent in eyes with good visual acuity, and so, the final vision would be better. Table 3 summarizes the BCVA change at 12 months after intravitreal anti-VEGF treatment of previous studies and current study [20,34-39,41].

In the present study, visual acuity was maintained or improved in $94.2 \%$ of cases at 12 months after treatment, which is higher than the $76.1 \%$ to $87.5 \%$ reported in previous studies on eyes with poor initial vision $[20,34,38]$. The CSMT significantly decreased until 12 months after treatment without major changes, similarly to the study on ranibizumab injection for PCV with good visual acuity [36]. As for PED, it decreased significantly at 12 months after treatment but held at $69.8 \%$. SRF showed the maximal improvement at 3 months after treatment and then increased again, but was improved significantly at all observation points. Lee et al. [20] and Mori et al. [36] also reported the greatest improvement of SRF at 3 months after treatment, presumably due to the effect of the three consecutive monthly injections, which protocol is common among all studies. After three loading doses in this study, the number of eyes with exudative changes was 18 (20.9\%). This is similar to the values reported by Mori et al. [36] (20\%, $10 /$ 50 eyes) who injected ranibizumab, and Lee et al. [20] (23.8\%) and Minami et al. [40] (20\%) who injected aflibercept.

Through subgroup analysis and multiple linear regression analysis, it was found that the factors affecting final BCVA were baseline BCVA and the presence of SRF at 3 months. Baseline CSMT did not affect the final BCVA. Previous studies have also shown that baseline BCVA independently affects posttreatment BCVA in PCV $[16,42,43]$. As mentioned above, good baseline BCVA indicates that the disease is relatively mild, so it is believed that the prognosis of visual acuity after treatment is expected to be good. The presence of SRF at 3 months means that the exudative change did not completely disappear even after three monthly loading doses, suggesting that the response to anti-VEGF treatment was poor.

The current study, due to its retrospective nature, did not strictly apply consistent retreatment criteria as typically are done in prospective studies. Moreover, since the sizes of PED and SRF were not measured, quantitative comparisons were not possible. Also, indocyanine green angiography was not performed in all patients at 12 months after treatment; thus, polyp closure was not consistently determined across the patient, even though unclosed polyps are thought to be the main cause of recurrence.

However, to the authors' best knowledge, this is the first long-term report of aflibercept treatment results for PCV with good visual acuity in Korea. In addition, it is a meaningful report of PCV sole disease excluding other diseases, and a reliable study with sample size of 86 . In conclusion, intravitreal aflibercept injections decreased CSMT and were effective in maintaining visual acuity in cases of PCV with good visual acuity, and the baseline good visual acuity independently affected final visual acuity. Therefore, early treatment is necessary to maintain good visual acuity in PCV with good baseline BCVA.

\section{Conflict of Interest}

No potential conflict of interest relevant to this article was reported.

\section{References}

1. Yannuzzi LA, Sorenson J, Spaide RF, Lipson B. Idiopathic polypoidal choroidal vasculopathy (IPCV). 1990. Retina 2012;32 Suppl 1:1-8.

2. Ciardella AP, Donsoff IM, Huang SJ, et al. Polypoidal choroidal vasculopathy. Surv Ophthalmol 2004;49:25-37.

3. Honda S, Matsumiya W, Negi A. Polypoidal choroidal vas- 
culopathy: clinical features and genetic predisposition. Ophthalmologica 2014;231:59-74.

4. Yoshimura N. Age-related macular degeneration in the Japanese. Nippon Ganka Gakkai Zasshi 2016;120:163-88.

5. Song SJ, Youm DJ, Chang Y, Yu HG. Age-related macular degeneration in a screened South Korean population: prevalence, risk factors, and subtypes. Ophthalmic Epidemiol 2009;16:304-10.

6. Park KH, Song SJ, Lee WK, et al. The results of nation-wide registry of age-related macular degeneration in Korea. J Korean Ophthalmol Soc 2010;51:516-23.

7. Bae K, Noh SR, Kang SW, et al. Angiographic subtypes of neovascular age-related macular degeneration in Korean: a new diagnostic challenge. Sci Rep 2019;9:9701.

8. Byeon SH, Lee SC, Oh HS, et al. Incidence and clinical patterns of polypoidal choroidal vasculopathy in Korean patients. Jpn J Ophthalmol 2008;52:57-62.

9. Uyama M, Matsubara T, Fukushima I, et al. Idiopathic polypoidal choroidal vasculopathy in Japanese patients. Arch Ophthalmol 1999;117:1035-42.

10. Uyama M, Wada M, Nagai Y, et al. Polypoidal choroidal vasculopathy: natural history. Am J Ophthalmol 2002;133:63948.

11. Lee WK, Kwon SI. Polypoidal choroidal vasculopathy. $J$ Korean Ophthalmol Soc 2000;41:2573-84.

12. Wong CW, Cheung CM, Mathur R, et al. Three-year results of polypoidal choroidal vasculopathy treated with photodynamic therapy: retrospective study and systematic review. Retina 2015;35:1577-93.

13. Nowak-Sliwinska P, van den Bergh H, Sickenberg M, Koh AH. Photodynamic therapy for polypoidal choroidal vasculopathy. Prog Retin Eye Res 2013;37:182-99.

14. Chen H, Liu K, Chen LJ, et al. Genetic associations in polypoidal choroidal vasculopathy: a systematic review and meta-analysis. Mol Vis 2012;18:816-29.

15. Kokame GT, Yeung L, Teramoto K, et al. Polypoidal choroidal vasculopathy exudation and hemorrhage: results of monthly ranibizumab therapy at one year. Ophthalmologica 2014;231:94-102.

16. Hikichi T, Higuchi M, Matsushita T, et al. Factors predictive of outcomes 1 year after 3 monthly ranibizumab injections and as-needed reinjections for polypoidal choroidal vasculopathy in Japanese patients. Retina 2013;33:1949-58.

17. Ogino K, Tsujikawa A, Yamashiro K, et al. Intravitreal injection of ranibizumab for recovery of macular function in eyes with subfoveal polypoidal choroidal vasculopathy. In- vest Ophthalmol Vis Sci 2013;54:3771-9.

18. Matsumiya W, Honda S, Kusuhara S, et al. Effectiveness of intravitreal ranibizumab in exudative age-related macular degeneration (AMD): comparison between typical neovascular AMD and polypoidal choroidal vasculopathy over a 1 year follow-up. BMC Ophthalmol 2013;13:10.

19. Koh A, Lai TY, Takahashi K, et al. Efficacy and safety of ranibizumab with or without verteporfin photodynamic therapy for polypoidal choroidal vasculopathy: a randomized clinical trial. JAMA Ophthalmol 2017;135:1206-13.

20. Lee JE, Shin JP, Kim HW, et al. Efficacy of fixed-dosing aflibercept for treating polypoidal choroidal vasculopathy: 1-year results of the VAULT study. Graefes Arch Clin Exp Ophthalmol 2017;255:493-502.

21. Oishi A, Kojima H, Mandai M, et al. Comparison of the effect of ranibizumab and verteporfin for polypoidal choroidal vasculopathy: 12-month LAPTOP study results. Am J Ophthalmol 2013;156:644-51.

22. Matsuoka M, Ogata N, Otsuji T, et al. Expression of pigment epithelium derived factor and vascular endothelial growth factor in choroidal neovascular membranes and polypoidal choroidal vasculopathy. $\mathrm{Br} J$ Ophthalmol 2004;88:809-15.

23. Tong JP, Chan WM, Liu DT, et al. Aqueous humor levels of vascular endothelial growth factor and pigment epithelium-derived factor in polypoidal choroidal vasculopathy and choroidal neovascularization. Am J Ophthalmol 2006;141:456-62.

24. Sasaki S, Miyazaki D, Miyake K, et al. Associations of IL23 with polypoidal choroidal vasculopathy. Invest Ophthalmol Vis Sci 2012;53:3424-30.

25. Zhao M, Bai Y, Xie W, et al. Interleukin-1ß level is increased in vitreous of patients with neovascular age-related macular degeneration (nAMD) and polypoidal choroidal vasculopathy (PCV). PLoS One 2015;10:e0125150.

26. Lo Giudice G, De Belvis V, Piermarocchi S, et al. Acute visual loss and chorioretinal infarction after photodynamic therapy combined with intravitreal triamcinolone. Eur $J$ Ophthalmol 2008;18:652-5.

27. Chan WM, Lam DS, Lai TY, et al. Photodynamic therapy with verteporfin for symptomatic polypoidal choroidal vasculopathy: one-year results of a prospective case series. Ophthalmology 2004;111:1576-84.

28. Akaza E, Yuzawa M, Matsumoto Y, et al. Role of photodynamic therapy in polypoidal choroidal vasculopathy. Jpn $J$ Ophthalmol 2007;51:270-7. 
29. Rosenfeld PJ, Brown DM, Heier JS, et al. Ranibizumab for neovascular age-related macular degeneration. $N$ Engl $J$ Med 2006;355:1419-31.

30. Brown DM, Kaiser PK, Michels M, et al. Ranibizumab versus verteporfin for neovascular age-related macular degeneration. N Engl J Med 2006;355:1432-44.

31. Kang HM, Koh HJ. Long-term visual outcome and prognostic factors after intravitreal ranibizumab injections for polypoidal choroidal vasculopathy. Am J Ophthalmol 2013;156:652-60.

32. Koh A, Lee WK, Chen LJ, et al. EVEREST study: efficacy and safety of verteporfin photodynamic therapy in combination with ranibizumab or alone versus ranibizumab monotherapy in patients with symptomatic macular polypoidal choroidal vasculopathy. Retina 2012;32:1453-64.

33. Papadopoulos N, Martin J, Ruan Q, et al. Binding and neutralization of vascular endothelial growth factor (VEGF) and related ligands by VEGF Trap, ranibizumab and bevacizumab. Angiogenesis 2012;15:171-85.

34. Cho HJ, Kim KM, Kim HS, et al. Intravitreal aflibercept and ranibizumab injections for polypoidal choroidal vasculopathy. Am J Ophthalmol 2016;165:1-6.

35. Saito M, Iida T, Kano M. Intravitreal ranibizumab for exudative age-related macular degeneration with good baseline visual acuity. Retina 2012;32:1250-9.

36. Mori R, Yuzawa M, Akaza E, Haruyama M. Treatment results at 1 year of ranibizumab therapy for polypoidal choroidal vasculopathy in eyes with good visual acuity. Jpn J Ophthalmol 2013;57:365-71.
37. Kato A, Yasukawa T, Suga K, et al. Intravitreal ranibizum$\mathrm{ab}$ for patients with neovascular age-related macular degeneration with good baseline visual acuity. Ophthalmologica 2015;233:27-34.

38. Kim YJ, Han SY, Kim JW, et al. Long-term treatment outcome of intravitreal aflibercept monotherapy for polypoidal choroidal vasculopathy. J Korean Ophthalmol Soc 2018;59:238-45.

39. Oshima Y, Kimoto K, Yoshida N, et al. One-year outcomes following intravitreal aflibercept for polypoidal choroidal vasculopathy in Japanese patients: the APOLLO Study. Ophthalmologica 2017;238:163-71.

40. Minami S, Nagai N, Suzuki M, et al. Benefits of aflibercept treatment for age-related macular degeneration patients with good best-corrected visual acuity at baseline. Sci Rep 2018;8:58.

41. Sakamoto S, Takahashi H, Inoue Y, et al. Intravitreal aflibercept for exudative age-related macular degeneration with good visual acuity: 2-year results of a prospective study. Clin Ophthalmol 2018;12:1137-47.

42. Gu X, Yu X, Dai H. Therapeutic effects of ranibizumab in patients with polypoidal choroidal vasculopathy. BMC Ophthalmol 2019;19:153.

43. Ho M, Lo EC, Young AL, Liu DT. Outcome of polypoidal choroidal vasculopathy at 1 year by combined therapy of photodynamic therapy with ranibizumab and predictive factors governing the outcome. Eye (Lond) 2014;28:146976. 